\title{
STRONG SOLUTIONS OF EVOLUTION EQUATIONS GOVERNED BY $m$-ACCRETIVE OPERATORS AND THE RADON-NIKODYM PROPERTY
}

\author{
ROBERT DEVILLE
}

(Communicated by Palle E. T. Jorgensen)

\begin{abstract}
We construct, in every Banach space which fails the Radon-Nikodym property, a nonlinear operator $A$ which is $m$-accretive for some equivalent norm in $X$, such that the domain of $A$ is not a singleton and such that the only strong solutions of the equation $u^{\prime}+A u \ni f$ are the constant ones.
\end{abstract}

\section{INTRODUCTION}

Let $X$ be a Banach space. An operator $A$ in $X$ is a map from $X$ into the power set $2^{X}$ of $X$. Let us denote

$$
D(A)=\{x \in X: A x \neq \varnothing\}
$$

the domain of $A$, and

$$
R(A)=\{y \in X: \exists x \in X \text { such that } y \in A x\}
$$

the range of $A$. We shall freely identify $A$ with the subset of $X \times X$ of all couples $(x, y)$ such that $x \in D(A)$ and $y \in A x$. The operator $A$ is called accretive if for every $(x, y)\left(x^{\prime}, y^{\prime}\right)$ in $A$ and for every $\lambda \geqq 0$ :

$$
\left\|x-x^{\prime}+\lambda\left(y-y^{\prime}\right)\right\| \geq\|x-y\| \text {. }
$$

The operator $A$ is said to be $m$-accretive if, moreover, for every $\lambda>0$

$$
R(I+\lambda A)=X,
$$

where $I$ stands for the identity operator in $X$. It is well known that $A$ is $m$-accretive if and only if $R(I+\lambda A)=X$ for some $\lambda>0$.

The operator $A$ is said to be trivial if there exists $a \in X$ such that $A=$ $\{a\} \times X$.

Now let us consider the equation:

$$
u^{\prime}+A u \ni f
$$

where $A$ is $m$-accretive and $f \in L^{1}(0, T ; X)$. We shall say that $u$ is a

Received by the editors February 5, 1990.

1980 Mathematics Subject Classification (1985 Revision). Primary 47H06; Secondary 46B22.

Key words and phrases. $m$-accretive operators, nonlinear evolution equations, Radon-Nikodym property, strong solutions. 
strong solution of (1) if $u$ is absolutely continuous and differentiable almost everywhere on $[0, T]$ and if

$$
u^{\prime}+A u(t) \ni f(t) \text { for almost every } t \in[0, T] .
$$

Observe that if $(x, y) \in A$ and $f(t)=y$ for almost every $t \in[0, T]$ then the function $u$ which is constant equal to $x$ is a strong solution of $(1)$ on $[0, T]$; in this case $u$ is called a constant solution of $(1)$ on $[0, T]$. We shall see below that in some cases the only strong solutions of $(1)$ on $[0, T]$ are the constant ones.

A Banach space $X$ has the Radon-Nikodym property if every bounded linear operator $T$ from $L^{1}([0,1])$ into $X$ is representable, which means that there exists $g \in L^{\infty}([0,1] ; X)$ such that for $f \in L^{1}([0,1]), T f=\int_{0}^{1} f(t) g(t) d t$. The classical Radon-Nikodym theorem expresses the fact that $\mathbf{R}$ has the RadonNikodym property. This property has been extensively studied in recent years (see e.g. [6] or [10]). Let us mention that every reflexive Banach space has the Radon-Nikodym property. On the other hand, $L^{1}\left(\mathbf{R}^{N}\right)$ and $B U C\left(\mathbf{R}^{N}\right)$ (the space of bounded uniformly continuous functions on $\mathbf{R}^{N}$ ) fail the RadonNikodym property.

Let us assume that $A$ is an $m$-accretive operator in a Banach space $X$. Then it is known that, if $u_{0}$ belongs to $D(A), f$ is in $B V(0, T ; X)$ and $X$ has the Radon-Nikodym property, the equation (1) has a unique strong solution on $[0, T]$ satisfying $u(0)=u_{0}$ (see [2] or [4]). It is also known that this result is no longer true in some concrete examples where $X$ fails the Radon-Nikodym property. For instance, let us consider the Hamilton-Jacobi equation in $\mathbf{R}^{N}$ :

$$
(H J) u_{t}+H(\nabla u)=f .
$$

Under a suitable hypothesis on $H$ we associate to this equation an $m$-accretive operator $A$ in $B U C\left(\mathbf{R}^{N}\right)$. However, even if the initial data $u_{0}$ is smooth $\left(u_{0} \in D(A)\right)$ and $f=0$, there is no strong solution of $(H J)$ on $[0, T]$ for every $T>0$. A similar remark holds for the nonlinear conservation laws in $\mathbf{R}^{N}$ to which one can associate an $m$-accretive operator in $L^{1}\left(\mathbf{R}^{N}\right)$ (see e.g. [2, $4,8])$.

In this note, we prove that in every Banach space which fails the RadonNikodym property, there exists a nontrivial operator $A$ in $X$, which is $m$ accretive for some equivalent norm in $X$, such that the only strong solutions of (1) on $[0, T]$ are the constant ones. In particular, if $f$ is not constant almost everywhere, or if $f$ is constant equal to $y \notin R(A)$ then equation (1) has no strong solution. The construction of the operator $A$ relies on the existence, for every Banach space $Y$ which fails the Radon-Nikodym property, of a Lipschitz function $\phi$ from $\mathbf{R}$ into $Y$ such that the set of all points of differentiability of $\phi$ is negligible. This last result will be proved in the appendix.

We also give various consequences of the existence of such an operator $A$, some of them having been already announced in [9]. 


\section{CONSTRUCTION OF AN $m$-ACCRETIVE OPERATOR}

In this section we prove

Theorem 2.1. Let $X$ be a Banach space. $X$ has the Radon-Nikodym property if and only if for every equivalent norm \|\| on $X$ and for every nontrivial $m$ accretive operator $A$ in $(X,\|\|)$ there exists $f \in L^{1}(0, T ; X)$ (where $T>0$ is arbitrary) such that (1) has a nonconstant strong solution on $[0, T]$.

We need the following elementary lemma whose proof is left to the reader. This is for instance an immediate consequence of Theorem 8-16 and of corollary of Theorem 8-6 of [11].

Lemma 2.2. Let $I$ be an interval of $\mathbf{R}$ and $f$ be an absolutely continuous function from I into $\mathbf{R}$. If $A=\left\{x \in I: f^{\prime}(x)=0\right\}$, then $\mu(f(A))=0$.

Proof of Theorem 2.1.

First, let assume that $X$ has the Radon-Nikodym property. Let \|\| be an equivalent norm on $X$ and $A$ be a nontrivial $m$-accretive operator in $(X,\|\|)$. Since $A$ is $m$-accretive, $D(A)$ is nonempty. Let us pick $a$ in $D(A)$. If $A a=X$ then, since $A$ is accretive $D(A)=\{a\}$ and so $A=\{a\} \times X$, which contradicts the assumption $A$ nontrivial. Hence, $A a \neq X$ and we can choose $b \in X \backslash A a$. Since $X$ has the Radon-Nikodym property, the theory of nonlinear semigroups (see e.g. [2] or [4]) tells us that the initial value problem:

$$
\left\{\begin{array}{l}
u^{\prime}+A u \ni b \\
u(0)=a
\end{array}\right.
$$

has a strong solution and this solution is nonconstant since $b \notin A a$.

Conversely, let $X$ be a Banach space which fails the Radon-Nikodym property. We shall construct an equivalent norm \|\| on $X$ and a nontrivial $m$-accretive operator $A$ in $(X,\|\|)$ such that for every $T>0$ and every $f \in L^{1}(0, T ; X)$, the only strong solutions of $u^{\prime}+A u \ni f$ are the constant ones.

Step 1. Construction of $A$. Let $|\cdot|$ be the original norm on $X, x_{0} \in X$ and $f \in X^{*}$ such that $f\left(x_{0}\right)=\left\|x_{0}\right\|=\|f\|=1$. Since $Y=\operatorname{Ker} f$ is a hyperplane of $X$ and $x_{0} \notin Y$, for every $z \in X$, there exists $a \in \mathbf{R}$ and $y \in Y$ such that $z=a x_{0}+y$, and we then define $\|z\|=\max (|a|,|y|)$. Clearly \|\| is an equivalent norm on $X$. By Corollary 3.3, there exists a Lipschitz function $\phi$ from $\mathbf{R}$ into $Y$ such that the set of all points of differentiability of $\phi$ is negligible. Without loss of generality, we can assume $\phi(0)=0$ and $\phi$ is 1-Lipschitz. Finally, define

$$
A=\left\{\left(t x_{0}+\phi(t), y\right): t \in \mathbf{R}, y \in Y\right\}
$$

Step 2. $A$ is $m$-accretive in $(X,\|\|)$. Let $(x, y),\left(x^{\prime}, y^{\prime}\right) \in A$ and $\lambda>0$. From the definition of $A$, there exists $t, t^{\prime} \in \mathbf{R}$ such that $x=t x_{0}+\phi(t)$, 


$$
\begin{aligned}
& x^{\prime}=t^{\prime} x_{0}+\phi\left(t^{\prime}\right) \text {, and } y, y^{\prime} \in Y \text {. Thus } \\
& \qquad \begin{aligned}
\left\|x-x^{\prime}\right\| & =\left\|\left(t-t^{\prime}\right) x_{0}+\phi(t)-\phi\left(t^{\prime}\right)\right\| \\
& =\sup \left(\left|t-t^{\prime}\right|,\left|\phi(t)-\phi\left(t^{\prime}\right)\right|\right)=\left|t-t^{\prime}\right|
\end{aligned}
\end{aligned}
$$

and

$$
\begin{aligned}
\left\|x-x^{\prime}+\lambda\left(y-y^{\prime}\right)\right\| & =\left\|\left(t-t^{\prime}\right) x_{0}+\phi(t)-\phi\left(t^{\prime}\right)+\lambda\left(y-y^{\prime}\right)\right\| \\
& =\sup \left(\left|t-t^{\prime}\right|,\left|\phi(t)-\phi\left(t^{\prime}\right)+\lambda\left(y-y^{\prime}\right)\right|\right) \\
& \geqq\left|t-t^{\prime}\right|,
\end{aligned}
$$

therefore, $A$ is accretive. On the other hand, since, for $\lambda>0$ :

$$
\begin{aligned}
I+\lambda A & =\left\{\left(t x_{0}+\phi(t), t x_{0}+\phi(t)+\lambda y\right): t \in \mathbf{R}, y \in Y\right\} \\
& =\left\{\left(t x_{0}+\phi(t), t x_{0}+y\right): t \in \mathbf{R}, y \in Y\right\}
\end{aligned}
$$

we have that, if $z=t x_{0}+y$ with $t \in \mathbf{R}$ and $y \in Y$, then $z \in(I+\lambda A)\left(t x_{0}+\phi(t)\right)$; thus $R(I+\lambda A)=X$ and $A$ is $m$-accretive.

Step 3 . The only strong solutions of (1) are the constant solutions. Let $u:[0, T] \rightarrow X$ be a strong solution of (1) that is not constant. Thus there exists $v \in \mathrm{AC}(0, T ; \mathbf{R})$ (the space of absolutely continuous functions from $[0, T]$ into $\mathbf{R})$ and $w \in \mathrm{AC}(0, T ; Y)$ such that $u(t)=v(t) x_{0}+w(t)$. Since for every $t \in[0, T], u(t) \in \overline{D(A)}=D(A)=\left\{t x_{0}+\phi(t): t \in \mathbf{R}\right\}$, we have $w(t)=\phi(v(t))$. Since $w(t)=u(t)-v(t) x_{0}$ for every $t \in[0, T]$ and since $u$ and $v$ are differentiable almost everywhere, $w$ is differentiable almost everywhere. On the other hand, if $B=\left\{t \in[0, T]\right.$ : either $v^{\prime}(t)$ does not exist or $\left.v^{\prime}(t)=0\right\}$ then by Lemma 2.2, $\mu(v(B))=0$. So, since $v$ is not constant, if $C=\left\{t \in[0, T]: v^{\prime}(t)\right.$ exists and $\left.v^{\prime}(t) \neq 0\right\}$, then $\mu(C) \neq 0$ and $\mu(v(C)) \neq 0$. Therefore the set

$$
A=\{t \in C: v(t) \text { is not a point of differentiability of } \phi\}
$$

is not negligible and we claim that $w$ is not differentiable at any point of $A$ which contradicts the fact that $w$ is differentiable almost everywhere. To prove our claim, let $t \in A$. Since for every $\varepsilon>0,\{v(t+h):|h|<\varepsilon\}$ is a neighborhood of $v(t)$ (use the continuity of $v$ and the fact that $v^{\prime}(t) \neq 0$ ), we have that

$$
\lim _{h \rightarrow 0} \frac{\phi(v(t+h))-\phi(v(t))}{v(t+h)-v(t)}
$$

does not exist. Now we write

$$
\frac{w(t+h)-w(t)}{h}=\frac{\phi(v(t+h))-\phi(v(t))}{v(t+h)-v(t)} \cdot \frac{v(t+h)-v(t)}{h} .
$$

This makes sense if $|h|$ is small enough (since then $v(t+h)-v(t) \neq 0$ ) and observing that $\lim _{h \rightarrow 0}(v(t+h)-v(t)) / h$ exists and is nonzero, we get that $\lim _{h \rightarrow 0}(w(t+h)-w(t)) / h$ does not exist, which proves our claim.

Remarks 2.3. 1. The idea of the proof of Theorem 2.1 is actually very simple: we have constructed an $m$-accretive operator $A$ in $(X,\|\|)$ such that the domain of $A$ is the graph of a Lipschitz function which is differentiable only 
on a set of Lebesgue measure zero. A solution of (1) is then forced to follow this graph and thus cannot be differentiable almost everywhere if it is not constant.

2. If we replace $A$ by $B$ :

$$
B=\left\{\left(t x_{0}+\phi(t),-x_{0}+y\right): t \in \mathbf{R}, y \in Y\right\}
$$

then $B$ is also $m$-accretive in $(X,\|\|)$ and the only strong solutions of $u^{\prime}+$ $B u \ni f$ are the constant solutions. And since $0 \notin R(B)$ the equation $u^{\prime}+B u \ni$ 0 has no strong solution: this result was obtained in [9]. Let us notice that since the equation $u^{\prime}+B u \ni 0$ always has at least one strong solution whenever $X$ has the Radon-Nikodym property and $B$ is $m$-accretive, we have obtained a new characterization of the Radon-Nikodym property.

3. Note that the Radon-Nikodym property is an isomorphic property i.e. does not depend on the equivalent norm on $X$. This justifies the fact that we have chosen an equivalent norm on $X$ in the statement of Theorem 2.1. In the linear case, the operators which are $m$-accretive for some equivalent norm are precisely the generators of uniformly bounded semigroups. The class of nonlinear operators which are $m$-accretive for some equivalent norm does not seem to be well known.

4. If $X$ is a Banach space, $A$ an $m$-accretive operator in $X, u_{0} \in \overline{D(A)}$ and $f \in L^{1}(0, T ; X)$, we say that $u \in \mathscr{C}(0, T ; X)$ is a weak solution of

$$
\left\{\begin{array}{l}
u^{\prime}+A u \ni f \\
u(0)=u_{0}
\end{array}\right.
$$

if there exists $u_{0, n} \in X, f_{n} \in L^{1}(0, T ; X)$, and $u_{n} \in \mathscr{C}(0, T ; X)$ such that $u_{n}$ is a strong solution of $u_{n}^{\prime}+A u_{n} \ni f_{n}$ on $[0, T]$ satisfying $u_{n}(0)=u_{0, n}$, $\lim _{n \rightarrow \infty} u_{0, n}=u_{0}$ in $X, \lim _{n \rightarrow \infty} f_{n}=f$ in $L^{1}(0, T ; X)$, and $\lim _{n \rightarrow \infty} u_{n}=u$ in $\mathscr{C}(0, T ; X)$. This notion of solution has been considered e.g. in [3]. If $A$ is the operator constructed in Theorem 2.1 , the only weak solution of $u^{\prime}+A u \ni f$ on $[0, T]$ are the constant solutions. Thus strong solutions, and more generally weak solutions, are not suitable to solve (2) in Banach spaces which fail the Radon-Nikodym property.

5. In order to solve (2) in a general Banach space $X$, one has to introduce the notion of mild solution: a function $u \in \mathscr{C}(0, T ; X)$ is called a mild solution of (2) on $[0, T]$ if for every $\varepsilon>0$, there exists $n \in \mathbf{N}, t_{0}=0<t_{1}<\cdots<$ $t_{n-1}<t_{n} \leqq T$ and $x_{0}, \ldots, x_{n}, y_{1}, \ldots, y_{n} \in X$ such that

$$
\begin{gathered}
\left(x_{i}-x_{i-1}\right) /\left(t_{i}-t_{i-1}\right)+A x_{i} \ni y_{i} \text { for } i=1, \ldots, n, \\
\left|t_{i}-t_{i-1}\right| \leqq \varepsilon \quad \text { for } i=1, \ldots, n, \sum_{i=1}^{n} \int_{t_{i-1}}^{t_{i}}\left|f(t)-y_{i}\right| d t \leqq \varepsilon, \\
\left|u_{0}-x_{0}\right| \leqq \varepsilon, \quad\left|u(t)-x_{i-1}\right| \leqq \varepsilon \text { for every } t \in\left[t_{i-1}-t_{i}\right], i=1, \ldots, n .
\end{gathered}
$$

It is known that (2) has a unique mild solution $u \in \mathscr{C}(0, T ; X)$ for every $u_{0} \in \overline{D(A)}$ and every $f \in L^{1}(0, T ; X)$. This shows existence and uniqueness 
of a solution of certain concrete partial differential equations (for instance, for the Hamilton-Jacobi equations which can be solved in $X=B U C\left(\mathbf{R}^{n}\right)$ or for the nonlinear conservation law equations which can be solved in $\left.X=L^{1}\left(\mathbf{R}^{N}\right)\right)$.

In a Banach space that has the Radon-Nikodym property, $u$ is a mild solution of $(2)$ on $[0, T]$ if and only if $u$ is a weak solution of $(2)$ on $[0, T]$. In a general Banach space, if $u$ is a weak solution of $(2)$ on $[0, T]$, then $u$ is a mild solution of $(2)$ on $[0, T]$. We refer the reader to [4] for these results. Remark 4, together with the above discussion, shows that in every Banach space which fails the Radon-Nikodym property, there exists an operator $A$ which is $m$-accretive for some equivalent norm in $X$, and a mild solution of (2) that is not a weak solution. Observe that in the example constructed in the proof of Theorem 2.1, if we set for $t \in[0, T], f(t)=g(t) x_{0}+h(t)$ with $g(t) \in \mathbf{R}$ and $h(t) \in Y$, the mild solution of $u^{\prime}+A u \ni f$ such that $u(0)=u_{0}$ is given by $u(t)=v(t) x_{0}+\phi(v(t))$ where $v$ satisfies $u(0)=v(0) x_{0}+\phi(v(0))$ and $v(t)-v(0)=\int_{0}^{t} g(t) d t$.

6. The operator $B$ as constructed in Theorem 2.1 is such that $B$ and $-B$ are $m$-accretive. It is easy to check that if $z=t x_{0}+y$ with $t \in \mathbf{R}$ and $y \in Y$, then if $\lambda>0$ :

$$
(I+\lambda A)^{-1}(z)=(t+\lambda) x_{0}+\phi(t+\lambda)
$$

thus for every $n \in \mathbf{N}$ :

$$
(I+\lambda A / n)^{-n}(z)=(t+\lambda) x_{0}+\phi(t+\lambda)
$$

and so, by the exponential formula, the mild solution of $u^{\prime}+B u \ni 0$ such that $u(0)=0$ is given by $u(t)=t x_{0}+\phi(t)$. Similarly, the mild solution of $v^{\prime}-B v \ni$ 0 such that $v(0)=T x_{0}+\phi(T)$ is given by $v(t)=(T-t) x_{0}+\phi(T-t)=u(T-t)$; thus we have time reversibility for the equation $u^{\prime}+B u \ni 0$. This phenomenon is not always true (see e.g. [5]). On the other hand, it is known that if $u$ is a weak solution of $u^{\prime}+A u \ni 0$ on $[0, T]$ then $v$ defined by $v(t)=u(T-t)$ is a weak solution of $v^{\prime}-A v \ni 0$ on $[0, T]$. In our example, $u$ and $v$ are mild solutions which are not weak solutions. So time reversibility for the equation $u^{\prime}+B u \ni 0$ does not force the solutions to be weak solutions.

\section{APPENDIX}

In this section, we construct, for every Banach space $X$ that fails the RadonNikodym property, a Lipschitz mapping $\phi: \mathbf{R} \rightarrow X$ which is almost nowhere differentiable. The following result is shown in [1]:

Proposition 3.1. Let $X$ be a Banach space. Then $X$ has the Radon-Nikodym property if and only if every Lipschitz function from $[0,1]$ into $X$ is differentiable almost everywhere.

Thus, if $X$ fails the Radon-Nikodym property, there exists a Lipschitz map $\phi:[0,1] \rightarrow X$ such that $\mu\left(N_{\phi}\right)>0$, where $\mu$ denotes the Lebesgue measure on $[0,1]$ and $N_{\phi}$ is the set of points of nondifferentiability of $\phi$. In this 
section we prove:

Theorem 3.2. Let $X$ be a Banach space which does not have the Radon-Nikodym property. Then there exists a Lipschitz mapping $\psi:[0,1] \rightarrow X$ such that $\mu\left(N_{\psi}\right)=1$.

Proof. Let $Y=\operatorname{Lip}([0,1], X)$ be the space of all Lipschitz mappings from $[0,1]$ into $X$ which vanish at zero. For $f \in Y$ let us define

$$
\|f\|_{Y}=\sup \left\{\frac{\|f(y)-f(y)\|}{y-x}: x, y \in[0,1], x \neq y\right\} .
$$

Then $\left(Y,\|\|_{Y}\right)$ is a Banach space. For $n \geqq 1$, let us consider the set

$$
U_{n}=\left\{f \in Y, \mu\left(N_{f}\right)>1-\frac{1}{n}\right\} .
$$

$U_{n}$ is open. Let $f \in U_{n}$ and $x \in[0,1]$. Let us denote

$$
D_{f}(x)=\lim _{\varepsilon \rightarrow 0} \operatorname{diam}\left\{\frac{f(x+h)-f(x)}{h}:|h|<\varepsilon\right\} \text {. }
$$

Since $x \in N_{f}$ if and only if $D_{f}(x)>0$, we have

$$
N_{f}=\bigcup\left\{A_{k}: k \geqq 1\right\}, \quad \text { where } A_{k}=\left\{x \in[0,1]: D_{f}(x) \geqq \frac{1}{k}\right\} .
$$

Since $\left(A_{k}\right)$ is increasing, there exists $k \geqq 1$ such that $\mu\left(A_{k}\right)>1-1 / n$. We claim that if $u \in Y$ and if $\|u\|_{Y}<1 / 2 k$ then $A_{k} \subseteq N_{f+u}$, thus showing that $f+u \in U_{n}$ and hence, that $U_{n}$ is open.

To prove the claim, it is enough to notice that for every $x \in[0,1]$ :

$$
\lim _{\varepsilon \rightarrow 0} \operatorname{diam}\left\{\frac{u(x+h)-u(x)}{h}:|h|<\varepsilon\right\}<\frac{1}{k} ;
$$

thus, for every $x \in A_{k}$ :

$$
\lim _{\varepsilon \rightarrow 0} \operatorname{diam}\left\{\frac{(f(x+h)+u(x+h))-(f(x)+u(x))}{h}:|h|<\varepsilon\right\}>0 .
$$

$U_{n}$ is dense. Let $x \in(0,1)$ be such that $\mu\left(I \cap N_{\phi}\right) / \mu(I)$ tends to 1 when the diameter of the interval $I$ containing $x$ tends to 0 . Let $I=[a, b]$ be an interval contained in $[0,1]$ and containing $x$ such that $\mu\left(I \cap N_{\phi}\right) / \mu(I)>$ $1-1 / 2 n$. The map $\psi$ defined by $\psi(t)=\phi(a+t(b-a))-\phi(a)$ belongs to $Y$ and $\mu\left(N_{\psi}\right)>1-1 / 2 n$. Let $f \in Y$. We claim that if $\alpha>0$ is small enough, then $f+\alpha \psi \in U_{n}$ which proves that $U_{n}$ is dense. Indeed, let $\gamma>0$ sufficiently small to have:

$$
\mu\left(\left\{x \in N_{f}: D_{f}(x)>\gamma\right\}\right)>\mu\left(N_{f}\right)-1 / 2 n .
$$

If we take $\alpha=\gamma /\|\psi\|_{Y}$, we get that:

$$
\begin{gathered}
N_{f+\alpha \psi} \supseteq\left([0,1] \backslash N_{f}\right) \cap N_{\psi}=A, \\
N_{f+\alpha \psi} \supseteq\left\{x \in N_{f}: D_{f}(x)>\gamma\right\}=B,
\end{gathered}
$$


and

$$
\mu(A \cup B)=\mu(A)+\mu(B)>\left(1-\mu\left(N_{f}\right)-\frac{1}{2 n}\right)+\left(\mu\left(N_{f}\right)-\frac{1}{2 n}\right)=1-\frac{1}{n} .
$$

Now, by Baire theorem, we have that $G=\bigcap_{n \in \mathbf{N}} U_{n}$ is a dense $\mathscr{G}_{\delta}$ in $Y$ and any $\psi \in G$ satisfies Theorem 3.2.

For proving Theorem 2.1, we have used the following consequence of Theorem 3.2.

Corollary 3.3. Let $X$ be a Banach space which fails the Radon-Nikodym property and $Y$ be a hyperplane of $X$. Then there exists a Lipschitz mapping $\phi: \mathbf{R} \rightarrow Y$ such that the set of all points of differentiability of $\phi$ is negligible.

Proof. Since $Y$ fails the Radon-Nikodym property, there exists by Theorem 2.2 a Lipschitz mapping $\psi:[0,1] \rightarrow Y$ such that $\mu\left(N_{\psi}\right)=1$. Now, if for $x \in \mathbf{R}$ we denote $[x]$ the greatest integer $\leqq x$, the function $\phi: \mathbf{R} \rightarrow Y$ defined by $\phi(x)=[x](\psi(1)-\psi(0))+\psi(x-[x])$ is Lipschitz continuous on $\mathbf{R}$ and satisfies Corollary 3.3.

Remark 3.4. If $X=L^{1}([0,1])$ the function $\phi:[0,1] \rightarrow X$ defined by $\phi(t)=$ $\mathbf{1}_{[0, T]}$ where $\mathbf{1}_{[0, T]}$ is the indicator function of $[0,1]$, is nowhere differentiable. The following improvement of Theorem 3.2 seems to be unknown:

If $X$ is a Banach space which does not have the Radon-Nikodym property, does there exist a Lipschitz map $\phi:[0,1] \rightarrow X$ which is nowhere differentiable?

\section{ACKNOWLEDGMENT}

The author wishes to thank Ph. Bénilan for stimulating discussions on the subject of this paper.

\section{REFERENCES}

1. W. Arendt, Vector valued Laplace transform and Cauchy problems, Israel J. Math. 59 (1987), 327-352.

2. Ph. Bénilan, Equation d'évolution dans un espace de Banach quelconque et applications, Thèse, Orsay, 1972.

3. Ph. Bénilan and $\mathrm{H}$. Brézis, Solutions faibles d'équations d'évolution dans un espace de Hilbert, Ann. Inst. Fourier 22 (1972), 311-319.

4. Ph. Bénilan, M. G. Crandall, and A. Pazy, Evolution problems governed by accretive operators (to appear).

5. __ Bonnes solutions d'un problème d'évolution semi-linéaire, C. R. Acad. Sci. Paris 306 (1988), 527-530.

6. R. Bourgin, Geometrical aspects of convex sets with the Radon-Nikodym property, Lecture Notes in Math., vol. 993, Springer-Verlag, 1983.

7. M. G. Crandall and T. Liggett, Generation of semi-groups on nonlinear transformation, Amer. J. Math. 11 (1971), 265-278.

8. M. G. Crandall and P. L. Lions, Viscosity solutions of Hamilton-Jacobi equations, Trans. Amer. Math. Soc. 277 (1983), 1-42.

9. R. Deville, Solutions fortes d'équations d'évolution et propriété de Radon-Nikodym, C. R. Acad. Sci. 309 (1989), 909-914.

10. J. Diestel and J. Uhl, Vector measures, Amer. Math. Soc., Providence, RI, 1977.

Laboratoire de Mathématiques, U. A. 741 CNRS, Université de Franche-Comté, 25030 Besancon Cedex, France 